\title{
Distribution of calretinin immunopositive somata and fibers in the rabbit midcingulate cortex
}

\author{
Mohi UDDIN ${ }^{1,2)}$ and Hideshi SHIBATA ${ }^{1,2) *}$ \\ 1)Laboratory of Veterinary Anatomy, Institute of Agriculture, Tokyo University of Agriculture and Technology, \\ Tokyo 183-8509, Japan \\ 2)United Graduate School of Veterinary Sciences, Gifu University, Gifu 501-1193, Japan
}

J. Vet. Med. Sci.

81(1): 57-65, 2019

doi: 10.1292/jvms.18-0577

Received: 1 October 2018

Accepted: 13 November 2018 Published online in J-STAGE:

23 November 2018

\begin{abstract}
The midcingulate cortex (MCC; area 24') resides in the mid-rostrocaudal part of the cingulate gyrus, and it plays important roles in nociceptive, cognitive and skeletomotor functions. The MCC has recently been shown to consist of four cortical areas (areas a24a', a24b', p24a' and p24b') in the rabbit, based on immunohistochemistry. To further characterize the organization of these areas, here we immunohistochemically identified structures immunopositive (+) for calretinin (CR) as a marker of a subpopulation of inhibitory neurons. CR+ somata were identified as multipolar and bipolar neurons. The multipolar neurons were predominant throughout the MCC. CR+ somata were present mainly in layer (L) $2 / 3$ and $L 6$, and $C R+$ fibers occurred mainly in $\mathrm{L} 1, \mathrm{~L} 2 / 3$ and L6. However, there were differences in the distribution of CR+ structures in each area. CR+ somata tended to be most densely distributed in area a24a', followed by area p24a', area a24b' and area p24b'. CR+ fibers were most densely distributed in area p24a', followed by area p24b', area a24a' and area a24b'. In addition, only areas p24a' and p24b' enclosed patchy CR+ fibers and terminals in deep L2/3. These results show the distinct distribution of CR+ structures in each area of the MCC in the rabbit, suggesting that $C R+$ neurons may contribute to information processing for cognitive functions in somewhat different manners in each area of the MCC.
\end{abstract}

KEY WORDS: areal distribution, calretinin, laminar distribution, midcingulate cortex, rabbit

The cingulate gyrus is located in the medial part of the cerebral cortex above the corpus callosum. The middle part of the cingulate gyrus along the anteroposterior axis is called the midcingulate cortex (MCC) or area $24^{\prime}$, which is well developed and highly differentiated in the rabbit [32]. A previous cytoarchitectural study with Nissl staining demonstrated that the rabbit MCC consists of two areas: area 24a' ventrally and area 24b' dorsally [32]. A later study based on the distribution of several neurochemical markers including calcium-binding protein parvalbumin (PV) suggested that area 24a' is subdivided into anterior area $a 24 a^{\prime}$ and posterior area p24a', and area $24 b^{\prime}$ is subdivided into anterior area a24b' and posterior area p24b' [34]. This parcellation pattern in the rabbit is more similar to that found in humans compared to the rodent parcellation [36, 38, 43].

The MCC is of great importance in human nociceptive, cognitive and skeletomotor functions [35]. Several studies in humans have shown that the anterior MCC (aMCC) is critically involved in avoidance behaviors [37], emotions such as anger and hostility $[7,23]$, and cognitive performance tasks such as response selection and error detection [2, 3, 27]. In contrast, the posterior MCC (pMCC) is involved in guiding body orientation and reflexive movements [37], and pain processing [19]. In altered conditions, aMCC is intimately linked to several diseases such as frontotemporal dementia [5] and obsessive-compulsive disorder [29], whereas pMCC is involved in progressive supranuclear palsy [5]. The differences between the aMCC and pMCC in functional involvement and susceptibility in human disorders heighten the need to develop experimental animal models for studies of physiology and disorder mechanisms linked to the human MCC.

The rabbit may be used as an experimental model for pathophysiological studies related to the human MCC, since its MCC parcellation pattern is more similar to that of humans than that of rodents as mentioned above. Although the structural organization has been studied with several neurochemical markers in the rabbit [34], more detailed studies are needed to characterize the similarities and differences in the distribution of other substances between each area of the MCC. Calretinin (CR) is a calciumbinding protein that is present in a subpopulation of inhibitory GABAergic neurons and is intimately linked to neuronal functions $[13,14,20,42]$. Moreover, CR is considered to be an excellent neurochemical substance to characterize the neocortical areas [1, 17]. Here, we selected CR as a neurochemical marker to further elucidate the structural organization of the rabbit MCC.

*Correspondence to: Shibata, H.: shibata@cc.tuat.ac.jp

O2019 The Japanese Society of Veterinary Science

This is an open-access article distributed under the terms of the Creative Commons Attribution Non-Commercial No Derivatives (by-nc-nd) License. (CC-BY-NC-ND 4.0: https://creativecommons.org/licenses/by-nc-nd/4.0/) 

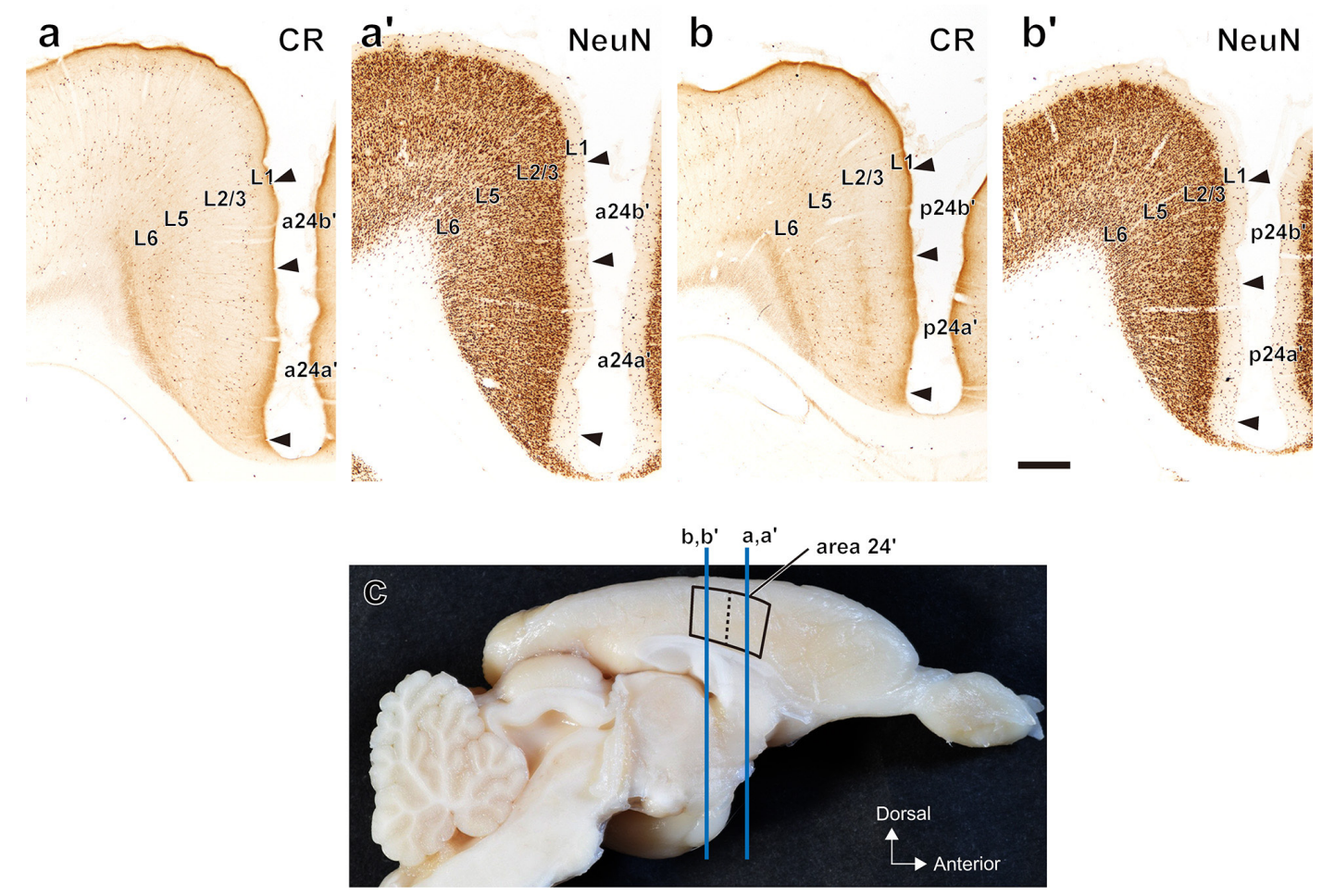

Fig. 1. The distribution of $\mathrm{CR}+$ structures $(\mathrm{a}, \mathrm{b})$ and the corresponding areal and laminar patterns demonstrated with NeuN immunostaining $\left(a^{\prime}, b^{\prime}\right)$ in the anterior $\left(a, a^{\prime}\right)$ and the posterior MCC $\left(b, b^{\prime}\right)$. (c) shows the location of the MCC (=area $\left.24^{\prime}\right)$ and the anteroposterior levels of the cutting planes of $a / a^{\prime}$ and $b / b^{\prime}$ (blue lines) in the medial view of the hemisphere. The dotted line shows the border between area a24' and area $24^{\prime}$. Arrowheads in $\mathrm{a}, \mathrm{a}^{\prime}, \mathrm{b}$ and $\mathrm{b}^{\prime}$ denote areal boundaries. Bar represents $500 \mu \mathrm{m}$, which is applicable to $\mathrm{a}, \mathrm{a}^{\prime}, \mathrm{b}$ and $\mathrm{b}^{\prime}$.

\section{MATERIALS AND METHODS}

All animal handling and experimental protocols were approved by the Ethics Committee of Animal Experimentation of Tokyo University of Agriculture and Technology (TUAT) and complied with the guideline for the care and use of laboratory animals at TUAT.

Five adult male New Zealand White rabbits $(2.35-2.70 \mathrm{~kg})$ were used. These animals were anesthetized with intramuscular injection of ketamine ( $50 \mathrm{mg} / \mathrm{kg}$ body weight) and intraperitoneal injection of pentobarbital sodium (60 mg/kg), and initially perfused through the ascending aorta with $1 l$ of $0.9 \% \mathrm{NaCl}$, followed by $2 l$ of $4 \%$ paraformaldehyde in $0.1 \mathrm{M}$ phosphate buffer ( $\mathrm{pH}$ 7.4). After perfusion, the brains were obtained from the skulls and placed in the same fixative at $4^{\circ} \mathrm{C}$ for $2-5 \mathrm{hr}$. The brains were cryoprotected in $30 \%$ sucrose in $0.1 \mathrm{M}$ phosphate buffer. Then, sections were cut on a freezing microtome at $50 \mu \mathrm{m}$ thickness in the coronal plane and divided into 5 series. Two of these series were used for the present study, while the remaining series were used for other studies.

One series of free-floating sections were dealt with a solution containing $0.3 \% \mathrm{H}_{2} \mathrm{O}_{2}$ and washed three times (5 min each) with Tris-HCl-buffered saline (TBS). After that, the sections were transferred to TBS containing 1\% normal horse serum (NHS) and $0.5 \%$ Triton-X-100 and incubated overnight at $4^{\circ} \mathrm{C}$. Next, the sections were incubated in TBS containing 1:2,000 or 8,000 mouse monoclonal antibody raised against CR (clone 6B8.2, Cat.\# MAB 1568, Merck Millipore, Darmstadt, Germany), 1\% NHS and $0.5 \%$ Triton-X-100 for $48 \mathrm{hr}$ at $4^{\circ} \mathrm{C}$. After incubation, the sections were rinsed with TBS and processed with a Vectastain Elite ABC kit (Vector Laboratories, Burlingame, CA, U.S.A.). Subsequently, the sections were washed with TBS and treated with $0.04 \%$ 3,3'-diaminobenzidine tetrahydrochloride and $0.01 \% \mathrm{H}_{2} \mathrm{O}_{2}$. After rinsing in TBS, the sections were mounted on slides, dried, defatted with a mixture of chloroform and ethanol (1:1), and coverslipped. Another series of sections were treated with 1:10,000 antibody against neuronal nuclear protein (NeuN) (Chemicon International, Temecula, CA, U.S.A.) with a similar immunohistochemical procedure for the identification of boundaries of each area and cortical layer of the MCC.

Sections were observed under an Eclipse-Ni photomicroscope (Nikon, Tokyo, Japan). Each area of the MCC was identified based on Vogt [34], and layers were divided into layer (L) 1, L2/3, L5 and L6 (Figs. 1 and 2). Neuronal types were identified based on somatodendritic morphology.

Images of selected sections were captured with a DS-Rildigital sight camera (Nikon) controlled with NIS-Elements (ver. 4.1) image analysis software (Nikon). The images were adjusted to attain optimal resolution, brightness, contrast and sharpness; trimmed with Adobe Photoshop CC (Adobe Systems, San Jose, CA, U.S.A.); and finally assembled into figures with Adobe Illustrator CC (Adobe Systems).

Semiquantitative evaluation was also performed in 8 sections with $200 \mu \mathrm{m}$-intervals in 3 individuals. The number of CR+ 

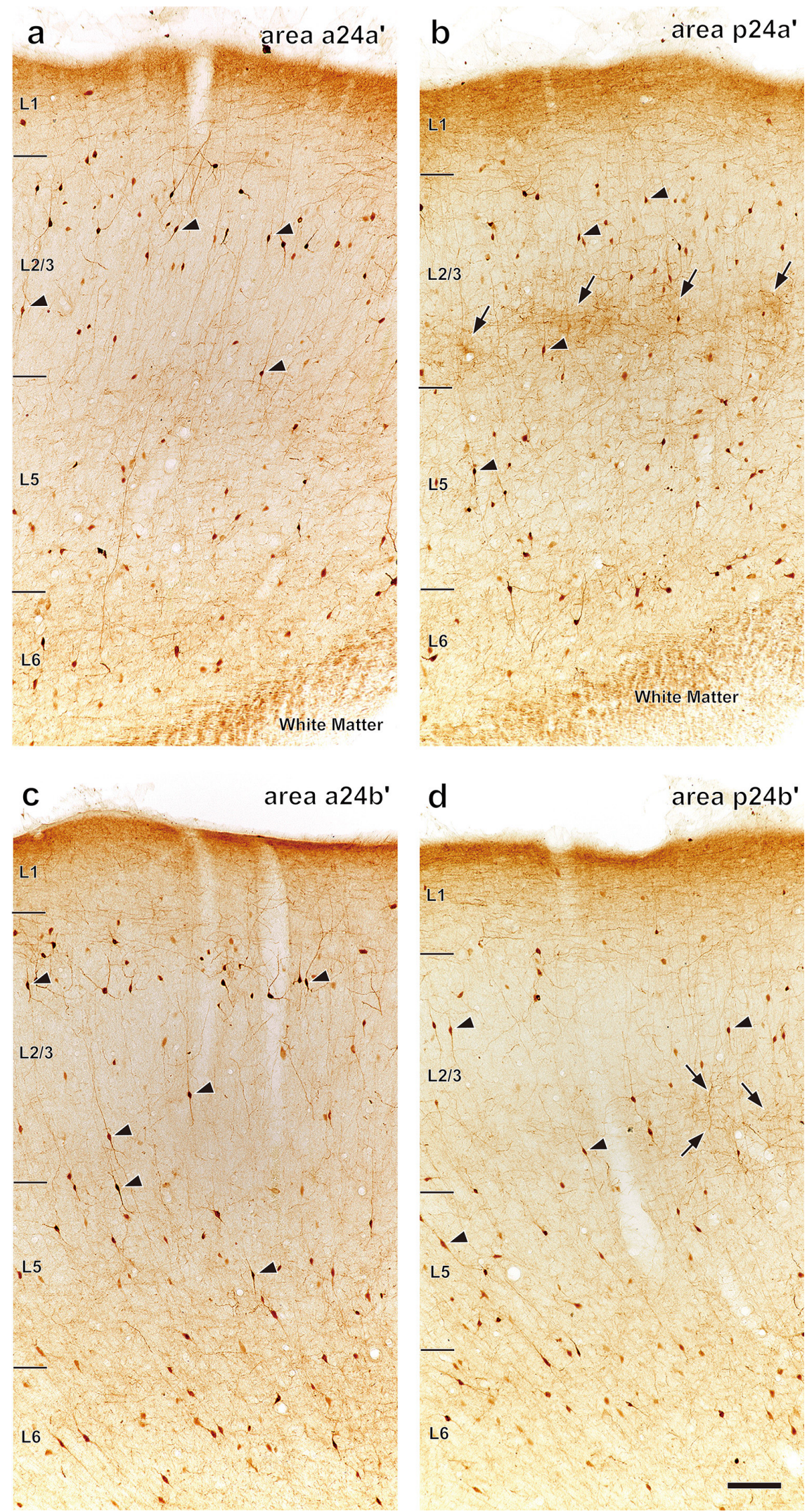

Fig. 2. $\quad$ CR + structures in the areas of the MCC. (a) area $a 24 a^{\prime}$, (b) area $p 24 a^{\prime}$, (c) area $a 24 b^{\prime}$ and (d) area p24b'. CR+ somata are more numerous in L2/3 and L6 than in L1 and L6. Most of CR+ somata are of the multipolar and some are of the bipolar type. Arrowheads denote examples of the bipolar somata. CR+ fibers are densely distributed in superficial L1. Furthermore, in areas p24a' and p24b', CR+ fibers are present in patches in deep L2/3 (arrows), whereas they are not present in areas a24a' and a24b'. Thin bars denote laminar borders and the thick bar in (d) represents $200 \mu \mathrm{m}$, which is applicable to (a)-(c). 

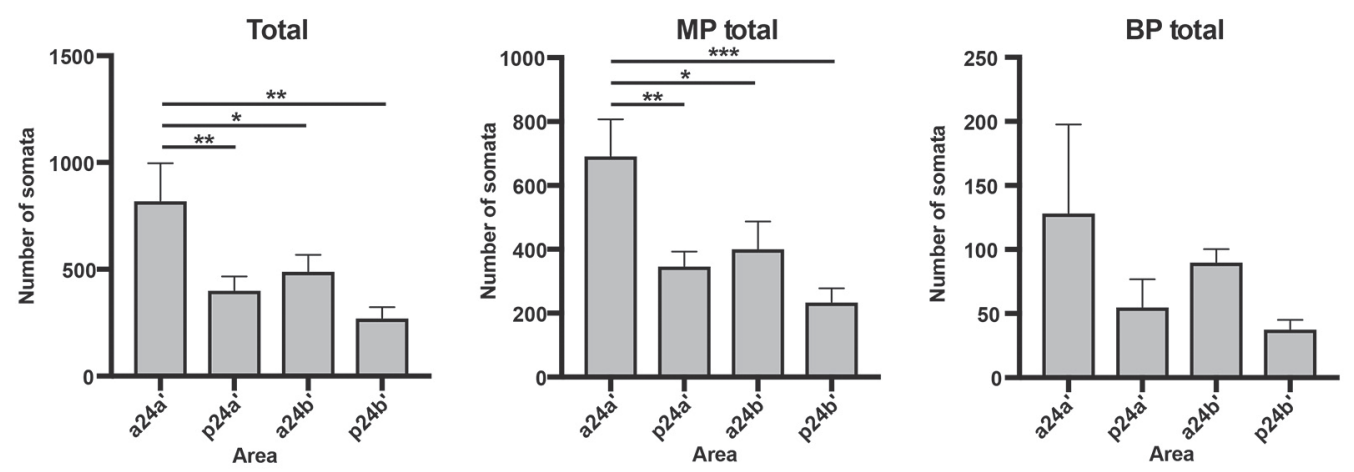

L1 total

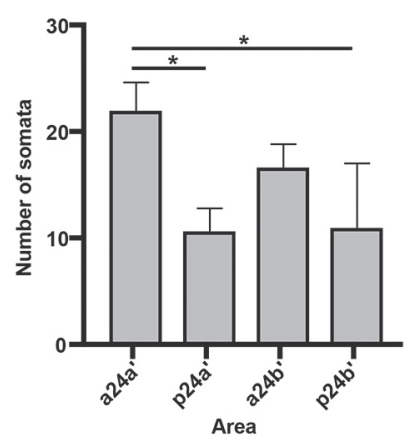

L2/3 total

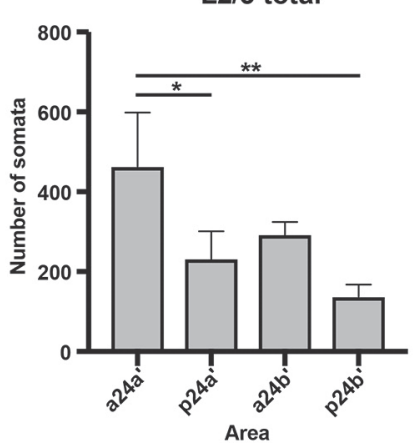

L5 total

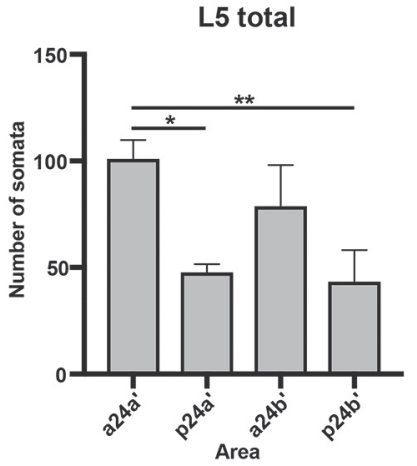

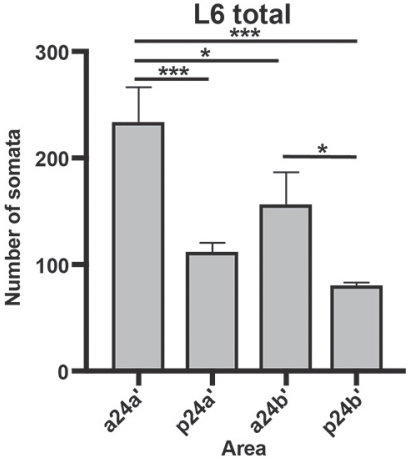

Fig. 3. Columnar graphs showing the statistical difference in number of $\mathrm{CR}+$ somata in each $\mathrm{MCC}$ area. *, $P<0.05$; **, $P<0.01$; and ***, $P<0.005$. $\mathrm{BP}=$ bipolar somata and $\mathrm{MP}=$ multipolar somata.

Table 1. Number (mean $\pm \mathrm{SD}$ ) of $\mathrm{CR}+$ somata in each area/layer of the MCC

\begin{tabular}{|c|c|c|c|c|c|c|c|c|c|c|c|c|}
\hline \multirow{2}{*}{ Layers } & \multicolumn{3}{|c|}{ area a24a' } & \multicolumn{3}{|c|}{ area p24a' } & \multicolumn{3}{|c|}{ area $\mathrm{a} 24 \mathrm{~b}^{\prime}$} & \multicolumn{3}{|c|}{ area $\mathrm{p} 24 \mathrm{~b}^{\prime}$} \\
\hline & MP & BP & All somata & MP & BP & All somata & MP & BP & All somata & MP & $\mathrm{BP}$ & All somata \\
\hline L1 & $22.0 \pm 2.6$ & $0.0 \pm 0.0$ & $22.0 \pm 2.6$ & $10.7 \pm 2.1$ & $0.0 \pm 0.0$ & $10.7 \pm 2.1$ & $16.7 \pm 2.1$ & $0.0 \pm 0.0$ & $16.7 \pm 2.1$ & $11.0 \pm 6.0$ & $0.0 \pm 0.0$ & $11.0 \pm 6.0$ \\
\hline $\mathrm{L} 2 / 3$ & $360.7 \pm 85.3$ & $103.0 \pm 56.8$ & $463.7 \pm 134.5$ & $184.3 \pm 53.0$ & $48.0 \pm 16.6$ & $232.3 \pm 68.7$ & $177.3 \pm 26.3$ & $62.0 \pm 9.6$ & $239.3 \pm 30.7$ & $108.3 \pm 23.2$ & $29.3 \pm 8.5$ & $137.7 \pm 30.0$ \\
\hline L5 & $86.7 \pm 6.8$ & $13.0 \pm 6.9$ & $99.7 \pm 11.1$ & $42.7 \pm 3.2$ & $5.3 \pm 3.1$ & $48.0 \pm 3.6$ & $61.0 \pm 23.1$ & $18.0 \pm 5.2$ & $79.0 \pm 19.0$ & $36.7 \pm 14.6$ & $7.0 \pm 6.6$ & $43.7 \pm 14.5$ \\
\hline L6 & $223.3 \pm 23.6$ & $11.0 \pm 8.9$ & $234.3 \pm 32.0$ & $110.7 \pm 10.2$ & $2.0 \pm 2.6$ & $112.7 \pm 7.6$ & $146.7 \pm 40.3$ & $10.3 \pm 10.7$ & $157.0 \pm 29.7$ & $79.3 \pm 1.5$ & $1.7 \pm 1.5$ & $81.0 \pm 2.0$ \\
\hline All layers & $692.7 \pm 114.7$ & $128.7 \pm 68.8$ & $821.3 \pm 175.6$ & $348.3 \pm 44.4$ & $55.3 \pm 21.4$ & $403.7 \pm 63.1$ & $401.7 \pm 85.1$ & $90.3 \pm 10.1$ & $492.0 \pm 76.5$ & $235.3 \pm 43.0$ & $38.0 \pm 7.0$ & $273.4 \pm 49.8$ \\
\hline
\end{tabular}

BP, bipolar, and MP, multipolar somata.

somata was counted with a $20 \times$ objective lens and the area of each layer of areas a24a', a24b', p24a' and p24b' was measured with NIS-Elements software. The data were compiled and the density of somata was calculated with Microsoft Excel 2016 (Microsoft, Redmond, WA, U.S.A.). The densities were statistically tested using ANOVA, followed by Bonferroni's multiple comparisons. $P<0.05$ was considered to be statistically significant.

\section{RESULTS}

\section{CR immunoreactivity in area $a 24 a^{\prime}$}

Area a24a' (Figs. 1a, 1a' and 2a) contained the greatest number of CR+ somata among all areas of the MCC (Fig. 3 and Table 1). Within this area, approximately $57 \%$ of the entire CR+ somata occurred in L2/3 and $29 \%$ in L6, whereas fewer CR+ somata were observed in L5 (12\%) and L1 (3\%) (Table 1). The number of CR+ somata in area a24a' was significantly higher than the other areas of the MCC (Fig. 3). The density of CR+ somata in this area tended to be higher than that in area p24a' and was significantly higher than those in area $a 24 b^{\prime}$ and area p24b' (Fig. 4 and Table 2). The difference in number was attributable mainly to that in the total number of multipolar somata and of somata of each layer (Fig. 3), whereas the difference in density was attributable mainly to that in the total density of multipolar and L2/3 somata (Fig. 4). In area a24a', the number and density were high in L2/3 and L6, but low in L1 and L5 (Tables 1 and 2). In CR+ somata, non-pyramidal multipolar neurons were predominant across all layers of the MCC (Figs. 2a, 5a and 5b, and Tables 1 and 2).

In L1 of area a24a', only a few CR+ multipolar neurons were observed (Figs. 2a, 5a and 5b, and Tables 1 and 2). In L2/3, $\mathrm{CR}+$ multipolar and bipolar neurons were mostly distributed in the superficial part, while in deep L2/3, fewer CR + somata were 

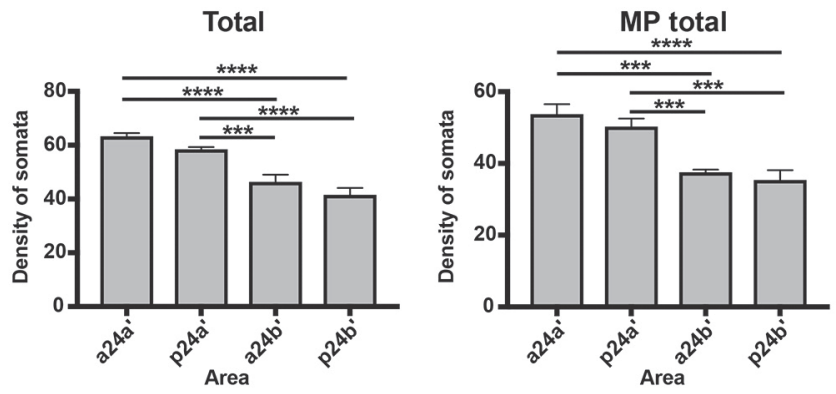

BP total
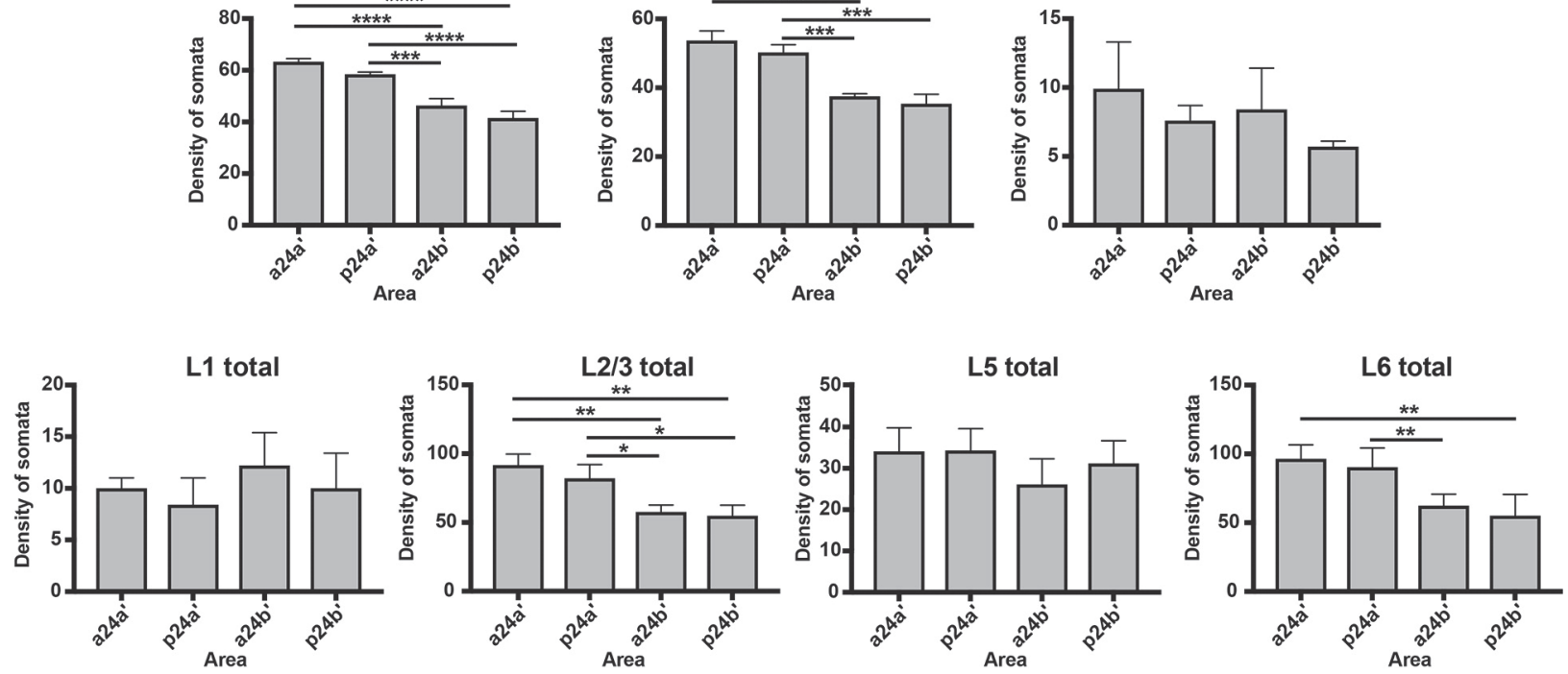

Fig. 4. Columnar graphs showing the statistical difference in density of $\mathrm{CR}+$ somata in each $\mathrm{MCC}$ area. *, $P<0.05$; **, $P<0.01 ; * * *$, $P<0.005$; and $* * * *, P<0.001$. $\mathrm{BP}=$ bipolar somata and $\mathrm{MP}=$ multipolar somata.

Table 2. Density $\left(n / \mathrm{mm}^{2}\right.$; mean $\left.\pm \mathrm{SD}\right)$ of $\mathrm{CR}+$ multipolar and bipolar somata in each area/layer of the MCC

\begin{tabular}{|c|c|c|c|c|c|c|c|c|c|c|c|c|}
\hline \multirow{2}{*}{ Layers } & \multicolumn{3}{|c|}{ Area a24a' } & \multicolumn{3}{|c|}{ Area p24a' } & \multicolumn{3}{|c|}{ Area a $24 b^{\prime}$} & \multicolumn{3}{|c|}{ Area p24b' } \\
\hline & MP & $\mathrm{BP}$ & All somata & MP & BP & All somata & MP & BP & All somata & MP & $\mathrm{BP}$ & All somata \\
\hline L1 & $10.0 \pm 1.0$ & 0 & $10.0 \pm 1.0$ & $8.4 \pm 3.2$ & 0 & $8.4 \pm 2.6$ & $12.2 \pm 3.1$ & 0 & $12.2 \pm 3.2$ & $10.0 \pm 3.0$ & 0 & $10.0 \pm 3.4$ \\
\hline $\mathrm{L} 2 / 3$ & $72.4 \pm 12.0$ & $19.3 \pm 4.2$ & $91.7 \pm 8.0$ & $66.0 \pm 9.1$ & $16.0 \pm 1.3$ & $82.0 \pm 10.0$ & $42.8 \pm 4.1$ & $14.7 \pm 2.2$ & $57.5 \pm 5.2$ & $42.6 \pm 6.1$ & $12.2 \pm 3.0$ & $54.8 \pm 7.8$ \\
\hline L5 & $29.1 \pm 1.2$ & $5.0 \pm 2.2$ & $34.1 \pm 5.7$ & $30.0 \pm 3.2$ & $4.3 \pm 2.0$ & $34.3 \pm 5.3$ & $23.9 \pm 2.2$ & $8.1 \pm 2.0$ & $26.1 \pm 6.2$ & $24.8 \pm 2.4$ & $6.4 \pm 2.1$ & $31.2 \pm 5.5$ \\
\hline L6 & $92.1 \pm 13.0$ & $4.3 \pm 3.1$ & $96.4 \pm 10.1$ & $88.1 \pm 16.0$ & $2.2 \pm 1.0$ & $90.3 \pm 14.0$ & $57.0 \pm 2.4$ & $5.3 \pm 2.0$ & $62.3 \pm 8.4$ & $53.0 \pm 13.7$ & $2.1 \pm 1.2$ & $55.1 \pm 15.4$ \\
\hline All layers & $53.7 \pm 2.8$ & $9.9 \pm 3.4$ & $63.6 \pm 1.2$ & $50.3 \pm 2.2$ & $7.6 \pm 1.1$ & $58.4 \pm 0.9$ & $37.5 \pm 0.8$ & $8.4 \pm 3.0$ & $46.3 \pm 2.7$ & $35.4 \pm 2.7$ & $5.7 \pm 0.4$ & $41.5 \pm 2.6$ \\
\hline
\end{tabular}

BP, bipolar, and MP, multipolar somata.

scattered (Fig. 2a). The multipolar neurons had oval, round or triangular somata with multiple dendrites directing various directions depending on each cell (Fig. 5a and 5b). The bipolar neurons had oval or fusiform somata and two vertically oriented dendritic arbors arising from the superficial and deep somatic poles (Fig. 5a). L5 and L6 contained mostly CR+ multipolar and a few bipolar somata (Fig. 2a, and Tables 1 and 2). The distribution of these types of neurons had laminar preference, but no areal preference across all areas of the MCC.

In area a24a', CR+ fibers were most abundant in L1 (Figs. 2a and 5c), followed by L6, L5 and L2/3 in decreasing order. CR+ fibers running almost rostrocaudally were also present in the cingulum, deep to L6 (Fig. 2a). In L1 of area a24a', most CR+ fibers coursed almost parallel to the pial surface in the superficial part to provide terminals to that part (Figs. $2 \mathrm{a}$ and $5 \mathrm{c}$ ). $\mathrm{CR}+$ dendritic processes also ran almost vertically in L1. In some cases, these processes were able to be traced to the multipolar and bipolar somata that were located mostly in L2/3. In L2/3, the majority of CR+ dendritic processes coursed vertically, and some showed a beaded appearance (Fig. 5b). In rare cases, the beaded processes were found to be issued from the multipolar or bipolar somata located mainly in L2/3 (Fig. 5b). In L5 and L6, CR+ fibers were directed randomly. In each of L2/3, L5 and L6, the distribution of $\mathrm{CR}+$ fibers was relatively uniform across the laminar thickness.

\section{CR immunoreactivity in area $p 24 a^{\prime}$}

In area $\mathrm{p} 24 \mathrm{a}^{\prime}$, the pattern of distribution of $\mathrm{CR}+$ somata was similar to that in area $\mathrm{a} 24 \mathrm{a}^{\prime}$, but the number of $\mathrm{CR}+$ somata in the total layers and each layer of area p24a' was significantly lower than that of area a24a' (Fig. 3 and Table 1). The density of CR+ somata in area p24a' tended to be lower than that in area a24a' and was significantly higher than that in areas a24b' and p24b' (Fig. 4 and Table 2). In L2/3 of area p24a', CR+ multipolar and bipolar somata were distributed throughout the thickness of L2/3 (Fig. 2b), unlike that of area a24a'.

Area p24a' enclosed more abundant CR+ dendrites, axons and terminals than area a24a' in all cortical layers (Figs. 2b and 5d), with a similar distribution pattern to area a24a'. Moreover, the deep part of L2/3 in area p24a' enclosed patchy CR+ fibers (arrows in Figs. $2 \mathrm{~b}$ and $5 \mathrm{e}$ ) that ran mostly parallel to the pial surface and provided axon terminals (Fig. 5f), while this feature was lacking in area a24a' (Fig. 2a). 

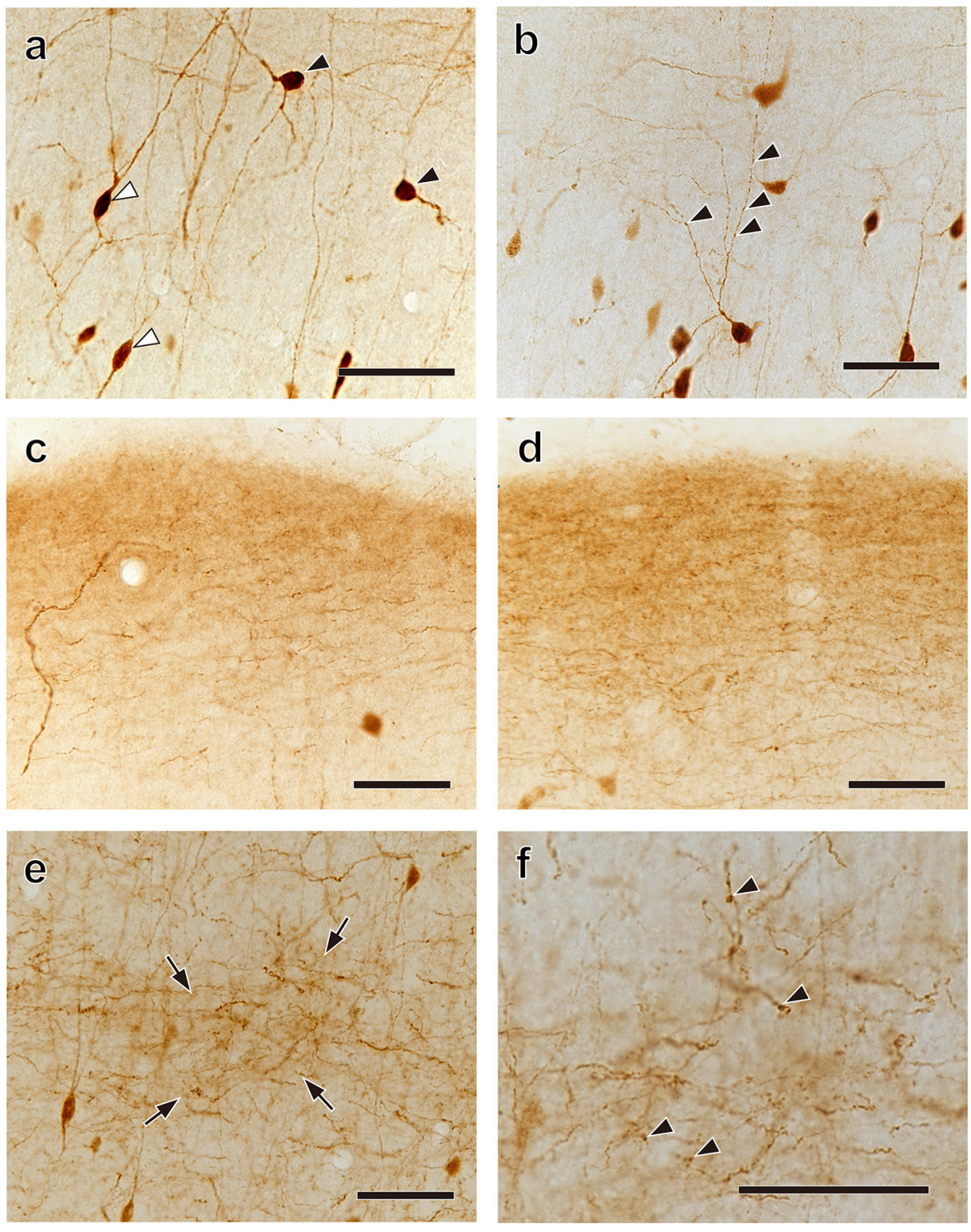

Fig. 5. (a) $\mathrm{CR}+$ multipolar (black arrowheads) and bipolar somata (white arrowheads) in superficial L2/3 of area a24a'. (b) Beaded dendritic processes (arrowheads) arising from a multipolar soma located in superficial L2/3 of area a24a'. (c) CR+ fibers in superficial L1 of area a24a'. A CR+ soma is also present in the deep part. (d) CR+ fibers in superficial L1 of area p24a'. These fibers are more abundant than those of area a24a'. (e) A patch of CR+ fibers and terminals (arrows) in deep L2/3 of area p24a'. (f) A higher view of part of the patch in (e), showing terminals (arrowheads). Bars represent $50 \mu \mathrm{m}$.

\section{CR immunoreactivity in area $a 24 b^{\prime}$}

In area $a 24 b^{\prime}$, the laminar distribution pattern of CR+ somata and fibers was again similar to those in area a24a' (Fig. 2). The total number and density of CR+ somata tended to be larger than those of area p24b' (Figs. 3 and 4, and Tables 1 and 2). Among the MCC areas, area a24b' generally contained the fewest CR+ fibers in all cortical layers (Fig. 2c).

\section{CR immunoreactivity in area $p 24 b^{\prime}$}

In area $\mathrm{p} 24 \mathrm{~b}^{\prime}$, the laminar distribution pattern of CR+ somata was similar to that in area p24a' (Fig. 2). Across all layers, the $\mathrm{CR}+$ somal number and density tended to be smallest in area p24b' compared to other areas of the MCC (Figs. 3 and 4, and Tables 1 and 2). Like area $24 \mathrm{a}^{\prime}$, patchy CR+ fibers with axon terminals occurred in deep L2/3, but this feature was less prominent than that of area p24a' (Fig. $2 b$ and $2 d$ ). 


\section{DISCUSSION}

The present study demonstrated that the distribution of CR+ somata and fibers in each area of the rabbit MCC differed from each other. Our findings greatly characterize each area of the MCC more than a previous study using another calcium-binding protein PV by Vogt [35], who showed only a tendency that area a24a' had fewer PV+ neurons than area $\mathrm{p} 24 \mathrm{a}^{\prime}$.

\section{Distributions of $C R+$ somata}

Most CR+ somata in the MCC are located in L2/3 and L6, while there are fewer in L5 and fewest in L1. This distribution pattern has not previously been reported in any cortical area of any other species. In the anterior cingulate cortex (ACC) of the cingulate gyrus, previous immunohistochemical studies have shown that $\mathrm{CR}+$ somata were observed mainly in L2/3 in the rat [10], cynomolgus monkey [8,9] and humans [11]. In the visual cortex, CR+ somata are also present mainly in L2/3 in the rabbit [25], mouse [24], hamster [21], dog [16] and cynomolgus monkey [22], whereas they are located in L1 and L2 in the bottlenose dolphin, L2/3 and L4a-c in humans [12], and L2/3, followed by L5, L6, L4 and L1 in decreasing order in the rat [13]. Although these studies show that neocortical areas tend to have numerous CR+ somata in L2/3 in many mammalian species, the rabbit MCC is characterized by the finding that L6 also has many $\mathrm{CR}+$ somata.

\section{Morphology of $C R+$ somata}

In the rabbit MCC, $\mathrm{CR}$ is expressed in non-pyramidal multipolar and bipolar neurons and the non-pyramidal multipolar neurons are predominant in any layer of any area. Although there have not been any similar previous studies in the MCC of other species, the present findings are in contrast to previous findings in the ACC of the cingulate gyrus, where bipolar and bitufted somata are predominant over multipolar somata in the rat [10], monkey [8] and humans [11]. In the visual cortex, it is also reported that bipolar somata are predominant over multipolar somata in the rabbit [25], mouse [24] and hamsters [21], whereas both types are even in the rat [13]. Therefore, although in the neocortical areas in most mammalian species studied, CR+ neurons are mainly of bipolar and bitufted neurons, the rabbit MCC is peculiar in that $\mathrm{CR}+$ neurons consist predominantly of the multipolar type.

\section{Distribution of $C R+$ fibers}

There is a tendency that $\mathrm{CR}+$ neuropil staining is more abundant posteriorly than anteriorly in the MCC. CR+ fibers were most abundant in L1, especially its superficial part, followed by L6, L5 and L2/3 in decreasing order. Deep L2/3 of areas p24a' and $\mathrm{p} 24 \mathrm{~b}^{\prime}$ also contains patchy $\mathrm{CR}+$ fibers, which are absent in areas a24a' and a $24 \mathrm{~b}^{\prime}$. These patterns of the distribution of CR+ neuropils have been demonstrated for the first time in the $\mathrm{MCC}$, and differ from the pattern in other regions of the cingulate gyrus in other mammalian species, where CR+ fibers are distributed homogeneously. For example, in the ACC of the rat, L1 was stained darker than the other layers, but no sublaminar preference of CR+ fibers was described within L1 [18]. In the ACC of monkeys and humans, the distribution of CR+ fibers were stained homogeneously across layers, except that deep L1 stained intensely in humans, but not so in monkeys [18].

Although there is no direct evidence for the existence of cells of origin of CR+ fibers observed in the MCC of the rabbit, previous hodological and immunohistochemical studies in this and other species may provide some clues to know the CR+ fiber architecture. As most CR+ neurons are a subpopulation of GABAergic neurons [14, 20], it is considered that most, if not all, $\mathrm{CR}+$ fibers in the MCC may be of intrinsic origins [4, 6]. Furthermore, given the existence of long-range GABAergic projections between cortical areas $[15,30,31]$, part of the CR+ fibers and terminals in L1 and L5 may correspond to those of the projections originating from retrosplenial area 30 to the MCC [39]. Other CR+ fibers and terminals that occur in patches in L2/3 of areas p24a' and $\mathrm{p} 24 \mathrm{~b}^{\prime}$ may also originate from within or outside of the MCC, but no previous studies have addressed this issue so far.

\section{Characterization of the rabbit $M C C$}

There are some differences in the $\mathrm{CR}+$ somata/fibers distribution in each area; $\mathrm{CR}+$ somata occur in superficial $\mathrm{L} 2 / 3$ of areas a24a' and a $24 b^{\prime}$, while they were distributed in entire L2/3 of areas p24a' and p24b'. There was a general tendency that CR+ somal density was highest in area $\mathrm{a} 24 \mathrm{a}^{\prime}$, followed by area $\mathrm{p} 24 \mathrm{a}^{\prime}$, area $\mathrm{a} 24 \mathrm{~b}^{\prime}$ and area $\mathrm{p} 24 \mathrm{~b}^{\prime}$ in decreasing order. CR+ fibers were most abundant in area $\mathrm{p} 24 \mathrm{a}^{\prime}$, followed by area $\mathrm{p} 24 \mathrm{~b}^{\prime}$, area $\mathrm{a} 24 \mathrm{a}^{\prime}$ and area $\mathrm{a} 24 \mathrm{~b}^{\prime}$ in decreasing order. These differences in the distribution of $\mathrm{CR}+$ somata and fibers suggest that each area may be anatomically distinct. Moreover, present findings support the anteroposterior and ventrodorsal parcellation of the rabbit MCC [34], which is similar to that of humans [33, 38, 43] and monkeys $[33,40]$. In rodents, the ventrodorsal parcellation of the MCC is noted $[26,35,36,41]$, whereas the anteroposterior parcellation is lacking $[35,36]$. Hence, the present study supports the notion that the MCC of humans is more similar to the MCC of rabbits than that of rodents. Although the distribution of $\mathrm{CR}+$ structures in the MCC has not been studied in humans so far, the relative similarity of the MCC parcellation pattern between the rabbit and humans underpins the idea that the rabbit may serve as a more suitable experimental model for pathophysiological studies of, at a minimum, parts of the human MCC than rodents.

\section{Functional significance}

$\mathrm{CR}+$ neurons are a subpopulation of inhibitory GABAergic neurons in the neocortex of the rat and mouse [13, 20, 42]. It is generally assumed that $\mathrm{CR}+$ multipolar neurons may provide information in a transcolumnar fashion in the cortex [4], whereas $\mathrm{CR}+$ bipolar neurons may integrate it within a columnar structure $[4,28]$. This may hold true for CR+ multipolar and bipolar neurons in the MCC of the rabbit. Since CR+ neurons occur not only in L2/3 as in other cingulate areas in rodents [10], but also in 
L6, and CR + multipolar neurons are distributed more densely than bipolar neurons, CR+ neurons in the MCC may be involved in more complex information processing by integrating signals within a column as well as receiving/sending signals from/to nearby columns. $\mathrm{CR}+$ fibers, some of which may be of extrinsic origins, may also modulate neuronal activity in the MCC. Therefore, in the rabbit MCC, $\mathrm{CR}+$ neurons and $\mathrm{CR}+$ fibers originating from inside and/or outside of the MCC may be involved in information processing in a more peculiar and complex way, in comparison with other cingulate cortical areas in rodents [10]. There may be some possible differences in information processing in each area of the MCC. This issue should be addressed in future studies.

ACKNOWLEDGMENTS. We gratefully acknowledge Dr. Yoshiko Honda at Tokyo Women's Medical University for her continuous encouragement during the course of this study, and our lab members, Tetsuhito Kigata and Ahmad Faisal Amiry, for their support and inspiration during the experiments.

\section{REFERENCES}

1. Barinka, F. and Druga, R. 2010. Calretinin expression in the mammalian neocortex: a review. Physiol. Res. 59: 665-677. [Medline]

2. Bush, G., Vogt, B. A., Holmes, J., Dale, A. M., Greve, D., Jenike, M. A. and Rosen, B. R. 2002. Dorsal anterior cingulate cortex: a role in rewardbased decision making. Proc. Natl. Acad. Sci. U.S.A. 99: 523-528. [Medline] [CrossRef]

3. Bush, G., Whalen, P. J., Rosen, B. R., Jenike, M. A., McInerney, S. C. and Rauch, S. L. 1998. The counting Stroop: an interference task specialized for functional neuroimaging — validation study with functional MRI. Hum. Brain Mapp. 6: 270-282. [Medline] [CrossRef]

4. Cauli, B., Zhou, X., Tricoire, L., Toussay, X. and Staiger, J. F. 2014. Revisiting enigmatic cortical calretinin-expressing interneurons. Front. Neuroanat. 8: 52. [Medline] [CrossRef]

5. Chiu, W. Z., Papma, J. M., de Koning, I., Donker Kaat, L., Seelaar, H., Reijs, A. E. M., Valkema, R., Hasan, D., Boon, A. J. W. and van Swieten, J. C. 2012. Midcingulate involvement in progressive supranuclear palsy and tau positive frontotemporal dementia. J. Neurol. Neurosurg. Psychiatry 83: 910-915. [Medline] [CrossRef]

6. DeFelipe, J. 1997. Types of neurons, synaptic connections and chemical characteristics of cells immunoreactive for calbindin-D28K, parvalbumin and calretinin in the neocortex. J. Chem. Neuroanat. 14: 1-19. [Medline] [CrossRef]

7. Dougherty, D. D., Shin, L. M., Alpert, N. M., Pitman, R. K., Orr, S. P., Lasko, M., Macklin, M. L., Fischman, A. J. and Rauch, S. L. 1999. Anger in healthy men: a PET study using script-driven imagery. Biol. Psychiatry 46: 466-472. [Medline] [CrossRef]

8. Gabbott, P. L. A. and Bacon, S. J. 1996. Local circuit neurons in the medial prefrontal cortex (areas 24a,b,c, 25 and 32) in the monkey: I. Cell morphology and morphometrics. J. Comp. Neurol. 364: 567-608. [Medline] [CrossRef]

9. Gabbott, P. L. A. and Bacon, S. J. 1996. Local circuit neurons in the medial prefrontal cortex (areas 24a,b,c, 25 and 32) in the monkey: II. Quantitative areal and laminar distributions. J. Comp. Neurol. 364: 609-636. [Medline] [CrossRef]

10. Gabbott, P. L. A., Dickie, B. G. M., Vaid, R. R., Headlam, A. J. N. and Bacon, S. J. 1997. Local-circuit neurones in the medial prefrontal cortex (areas 25, 32 and 24b) in the rat: morphology and quantitative distribution. J. Comp. Neurol. 377: 465-499. [Medline] [CrossRef]

11. Gabbott, P. L. A., Jays, P. R. L. and Bacon, S. J. 1997. Calretinin neurons in human medial prefrontal cortex (areas 24a,b,c, 32', and 25). J. Comp. Neurol. 381: 389-410. [Medline] [CrossRef]

12. Glezer, I. I., Hof, P. R. and Morgane, P. J. 1992. Calretinin-immunoreactive neurons in the primary visual cortex of dolphin and human brains. Brain Res. 595: 181-188. [Medline] [CrossRef]

13. Gonchar, Y. and Burkhalter, A. 1997. Three distinct families of GABAergic neurons in rat visual cortex. Cereb. Cortex 7: 347-358. [Medline] [CrossRef]

14. Gonchar, Y., Wang, Q. and Burkhalter, A. 2008. Multiple distinct subtypes of GABAergic neurons in mouse visual cortex identified by triple immunostaining. Front. Neuroanat. 1: 3. [Medline] [CrossRef]

15. Higo, S., Udaka, N. and Tamamaki, N. 2007. Long-range GABAergic projection neurons in the cat neocortex. J. Comp. Neurol. 503: 421-431. [Medline] [CrossRef]

16. Hof, P. R., Bogaert, Y. E., Rosenthal, R. E. and Fiskum, G. 1996. Distribution of neuronal populations containing neurofilament protein and calcium-binding proteins in the canine neocortex: regional analysis and cell typology. J. Chem. Neuroanat. 11: 81-98. [Medline] [CrossRef]

17. Hof, P. R., Glezer, I. I., Condé, F., Flagg, R. A., Rubin, M. B., Nimchinsky, E. A. and Vogt Weisenhorn, D. M. 1999. Cellular distribution of the calcium-binding proteins parvalbumin, calbindin, and calretinin in the neocortex of mammals: phylogenetic and developmental patterns. J. Chem. Neuroanat. 16: 77-116. [Medline] [CrossRef]

18. Hof, P. R., Lüth, H.J., Rogers, J. H. and Celio, M. R. 1993. Calcium-binding proteins define subpopulations of interneurons in cingulate cortex. pp. 181-205. In: Neurobiology of Cingulate Cortex and Limbic Thalamus (Vogt, B. A. and Gabriel. M. eds.), Birkhäuser, Boston.

19. Kisler, L. B., Granovsky, Y., Sinai, A., Sprecher, E., Shamay-Tsoory, S. and Weissman-Fogel, I. 2016. Sex dimorphism in a mediatory role of the posterior midcingulate cortex in the association between anxiety and pain sensitivity. Exp. Brain Res. 234: 3119-3131. [Medline] [CrossRef]

20. Kubota, Y., Hattori, R. and Yui, Y. 1994. Three distinct subpopulations of GABAergic neurons in rat frontal agranular cortex. Brain Res. 649: 159-173. [Medline] [CrossRef]

21. Lee, J. E., Ahn, C. H., Lee, J. Y., Chung, E. S. and Jeon, C. J. 2004. Nitric oxide synthase and calcium-binding protein-containing neurons in the hamster visual cortex. Mol. Cells 18: 30-39. [Medline] [CrossRef]

22. Meskenaite, V. 1997. Calretinin-immunoreactive local circuit neurons in area 17 of the cynomolgus monkey, Macaca fascicularis. J. Comp. Neurol. 379: 113-132. [Medline] [CrossRef]

23. Nakagawa, S., Takeuchi, H., Taki, Y., Nouchi, R., Sekiguchi, A., Kotozaki, Y., Miyauchi, C. M., Iizuka, K., Yokoyama, R., Shinada, T., Yamamoto, Y., Hanawa, S., Araki, T., Hashizume, H., Kunitoki, K., Sassa, Y. and Kawashima, R. 2017. The anterior midcingulate cortex as a neural node underlying hostility in young adults. Brain Struct. Funct. 222: 61-70. [Medline] [CrossRef]

24. Park, H. J., Kong, J. H., Kang, Y. S., Park, W. M., Jeong, S. A., Park, S. M., Lim, J. K. and Jeon, C. J. 2002. The distribution and morphology of calbindin D28K- and calretinin-immunoreactive neurons in the visual cortex of mouse. Mol. Cells 14: 143-149. [Medline]

25. Park, H. J., Lee, S. N., Lim, H. R., Kong, J. H. and Jeon, C. J. 2000. Calcium-binding proteins calbindin D28K, calretinin, and parvalbumin immunoreactivity in the rabbit visual cortex. Mol. Cells 10: 206-212. [Medline] [CrossRef]

26. Paxinos, G., Watson, C., Carrive, P., Kirkcaldie, M. and Ashwell, K. W. S. 2009. Chemoarchitectonic Atlas of the Rat Brain, 2nd ed., Elsevier, Amsterdam. 
27. Pereira, M. G., de Oliveira, L., Erthal, F. S., Joffily, M., Mocaiber, I. F., Volchan, E. and Pessoa, L. 2010. Emotion affects action: Midcingulate cortex as a pivotal node of interaction between negative emotion and motor signals. Cogn. Affect. Behav. Neurosci. 10: 94-106. [Medline] [CrossRef]

28. Raghanti, M. A., Spocter, M. A., Butti, C., Hof, P. R. and Sherwood, C. C. 2010. A comparative perspective on minicolumns and inhibitory GABAergic interneurons in the neocortex. Front. Neuroanat. 4: 3. [Medline]

29. Saxena, S., O’Neill, J. and Rauch, S. L. 2009. The role of cingulate cortex dysfunction in obsessive-compulsive disorder. pp. 588-617. In: Cingulate Neurobiology and Disease (Vogt, B. A. ed.), Oxford University Press, London.

30. Tamamaki, N. and Tomioka, R. 2010. Long-range GABAergic connections distributed throughout the neocortex and their possible function. Front. Neurosci. 4: 202. [Medline] [CrossRef]

31. Tomioka, R., Okamoto, K., Furuta, T., Fujiyama, F., Iwasato, T., Yanagawa, Y., Obata, K., Kaneko, T. and Tamamaki, N. 2005. Demonstration of long-range GABAergic connections distributed throughout the mouse neocortex. Eur. J. Neurosci. 21: 1587-1600. [Medline] [CrossRef]

32. Vogt, B. A. 1993. Structural organization of cingulate cortex: areas, neurons, and somatodendritic transmitter receptors. pp. 19-70. In: Neurobiology of Cingulate Cortex and Limbic Thalamus (Vogt, B. A. and Gabriel. M. eds.), Birkhäuser, Boston.

33. Vogt, B. A. 2009. Architecture, neurocytology and comparative organization of monkey and human cingulate cortices. pp. 65-91. In: Cingulate Neurobiology and Disease (Vogt, B. A. ed.), Oxford University Press, New York.

34. Vogt, B. A. 2016. Cytoarchitecture and neurocytology of rabbit cingulate cortex. Brain Struct. Funct. 221: 3571-3589. [Medline] [CrossRef]

35. Vogt, B. A. 2016. Midcingulate cortex: Structure, connections, homologies, functions and diseases. J. Chem. Neuroanat. 74: 28-46. [Medline] [CrossRef]

36. Vogt, B. A. and Paxinos, G. 2014. Cytoarchitecture of mouse and rat cingulate cortex with human homologies. Brain Struct. Funct. 219: 185-192. [Medline] [CrossRef]

37. Vogt, B. A. and Sikes, R. W. 2009. Cingulate nociceptive circuitry and roles in pain processing: The cingulate premotor pain model. pp. 331-333. In: Cingulate Neurobiology and Disease (Vogt, B. A. ed.), Oxford University Press, New York.

38. Vogt, B. A., Berger, G. R. and Derbyshire, S. W. G. 2003. Structural and functional dichotomy of human midcingulate cortex. Eur. J. Neurosci. 18: 3134-3144. [Medline] [CrossRef]

39. Vogt, B. A., Sikes, R. W., Swadlow, H. A. and Weyand, T. G. 1986. Rabbit cingulate cortex: cytoarchitecture, physiological border with visual cortex, and afferent cortical connections of visual, motor, postsubicular, and intracingulate origin. J. Comp. Neurol. 248: 74-94. [Medline] [CrossRef]

40. Vogt, B. A., Vogt, L., Farber, N. B. and Bush, G. 2005. Architecture and neurocytology of monkey cingulate gyrus. J. Comp. Neurol. 485: 218-239. [Medline] [CrossRef]

41. Watson, C. and Paxinos, G. 2010. Chemoarchitectonic Atlas of the Mouse Brain, Elsevier, Amsterdam.

42. Xu, X., Roby, K. D. and Callaway, E. M. 2010. Immunochemical characterization of inhibitory mouse cortical neurons: three chemically distinct classes of inhibitory cells. J. Comp. Neurol. 518: 389-404. [Medline] [CrossRef]

43. Yu, C., Zhou, Y., Liu, Y., Jiang, T., Dong, H., Zhang, Y. and Walter, M. 2011. Functional segregation of the human cingulate cortex is confirmed by functional connectivity based neuroanatomical parcellation. Neuroimage 54: 2571-2581. [Medline] [CrossRef] 